\title{
A Summary of Phenotypes Observed in the In Vivo Rodent Alpha-Synuclein Preformed Fibril Model
}

\author{
Nicole K. Polinski* \\ The Michael J. Fox Foundation for Parkinson's Research, New York, NY, USA \\ Accepted 9 August 2021 \\ Pre-press 2 September 2021
}

\begin{abstract}
The use of wildtype recombinant alpha-synuclein preformed fibrils (aSyn PFFs) to induce endogenous alphasynuclein to form pathological phosphorylation and trigger neurodegeneration is a popular model for studying Parkinson's disease (PD) biology and testing therapeutic strategies. The strengths of this model lie in its ability to recapitulate the phosphorylation/aggregation of aSyn and nigrostriatal degeneration seen in $\mathrm{PD}$, as well as its suitability for studying the progressive nature of PD and the spread of aSyn pathology. Although the model is commonly used and has been adopted by many labs, variability in observed phenotypes exists. Here we provide summaries of the study design and reported phenotypes from published reports characterizing the aSyn PFF in vivo model in rodents following injection into the brain, gut, muscle, vein, peritoneum, and eye. These summaries are designed to facilitate an introduction to the use of aSyn PFFs to generate a rodent model of PD—highlighting phenotypes observed in papers that set out to thoroughly characterize the model. This information will hopefully improve the understanding of this model and clarify when the aSyn PFF model may be an appropriate choice for one's research.
\end{abstract}

Keywords: Alpha-synuclein, Parkinson disease, preformed fibril, model

\section{INTRODUCTION}

Parkinson's disease (PD) is a neurodegenerative disorder affecting approximately $1 \%$ of the population over the age of 60 . Characterized by motor disturbances as well as non-motor symptoms, the pathology of PD involves deposits of aggregated, phosphorylated alpha-synuclein (aSyn) protein in affected tissues and brain structures and degeneration of dopaminergic neurons in the substantia nigra pars compacta (SNpc). Given that PD is a human-specific condition, various models have been developed to enable research and therapeutic development for this

\footnotetext{
*Correspondence to: Nicole K. Polinski, Grand Central Station, PO Box 4777, New York, NY 10163, USA. Tel.: +1 2125090995 /Ex375; E-mail: npolinski@michaeljfox.org.
}

disease. Common models include injection of neurotoxins to trigger degeneration of the dopaminergic neurons of the SNpc, transgenic rodent models carrying PD-related genetic mutations, and induction of aSyn pathology through viral vector-mediated overexpression of aSyn, among others [1-3]. All models present with advantages and disadvantages, so selection of the model should be based on the desired pathology for the intended research question.

In the last 10 years, a model has arisen that capitalizes on the observations made by Braak and colleagues that aSyn pathology progressively accumulates in different brain regions following a spatiotemporal pattern that suggests spreading [4-7]. This model, dubbed the aSyn preformed fibril (PFF) model, uses injection of recombinant aSyn protein that has been stimulated to form aggregates and son- 
icated to produce short fibrils [8-10]. These aSyn PFFs cause templating of endogenous aSyn into pathological species characterized by phosphorylation at S129 (pS129 aSyn), beta-sheet formation, and aggregation, followed by increases in autophagy and neuronal dysfunction [11]. The flexibility of this model allows injection of different forms of aSyn PFFs (e.g., mouse vs. human aSyn, mutated aSyn), unilateral or bilateral injection, targeting of different brain regions and administration through different peripheral routes to model distinct aspects of the disease. This flexibility is a strength of the model but also serves as a weakness, as the distinct protocols lead to different pathologies which has hampered crossstudy comparisons. To better understand the various study designs employed for the aSyn PFF model and the resulting pathologies, a survey of the literature was performed and is summarized within this manuscript.

\section{GUIDE TO READING AND INTERPRETING THE TABLES}

As hundreds of studies using the aSyn PFF model have been published, Tables 1-9 herein contain information specifically from publications that sought to phenotype the effects of injection of recombinant wildtype aSyn PFFs into rodents to develop a PD model. As a result, the tables are not comprehensive in nature but do contain reports from a variety of studies across laboratories.

Studies focusing on the uptake of aSyn following injection have been excluded as the study is not designed to thoroughly assess resulting pathology. Studies using the aSyn PFF model to test the effect of an intervention have been excluded as the focus is on the therapeutic intervention tested rather than the characterization of the pathological process and timelines. Studies injecting aSyn PFFs to model another disease (e.g., Multiple System Atrophy) were excluded to focus specifically on PD. Studies injecting aSyn PFFs into non-human primates or using aSyn PFFs in cell culture were excluded for the sake of focus. Studies injecting rodent/patient brainderived material were excluded due to concerns that the injectate is not homogenous and the concentration of aSyn and other protein components cannot be known or compared across studies. Although a number of studies have been published analyzing the differences in pathogenicity of fibrils of different conformations [12-18], different aSyn mutations
[19-22], different aSyn truncations [23-25], and different aSyn post-translational modifications [26], these were excluded from the summary tables as the objective of these experiments is to compare pathogenicity relative to wildtype aSyn PFFs and therefore the nuanced information requires a different venue.

Tables 1-9 are organized by categories such as: injected species (mouse vs. rat), route of administration of aSyn PFFs, and species of aSyn PFF (human vs. mouse). To understand the variation in observed phenotypes within the model, readers should compare only within categories rather than across categories. Please note that there may be differences in study design within categories (e.g., unilateral vs. bilateral injection, wildtype vs. transgenic rodent) that should be taken into account when drawing conclusions on timelines and robustness of phenotypes.

Papers included within the tables are organized chronologically, with high-level information on study design, outcome measures, and notes that may provide additional context for the reader. Information on study design includes the rodent strain used, the injectate, the dose of aSyn PFFs with information on whether this dose was administered bilaterally or unilaterally (for bilateral injections, the total dose noted was for each hemisphere), and the days postinjection (DPI) at which time the model was analyzed. Reported phenotypes are separated by category to facilitate comparisons of common readouts across studies. The time post-injection at which the phenotype was observed is included, with a "+" indicating the phenotype was also observed at the later timepoints. If later timepoints were analyzed within the study but the "+" sign is absent, this indicates that either the phenotype was not analyzed at the later timepoints or was analyzed but not observed. If a phenotype was observed in a particular structure, the structure is included in parentheses. Readouts that were not included in the study are denoted as "N/A". Please note, to fully understand all reported or absent phenotypes in the models, a separate literature review is required.

\section{SUMMARY OF PHENOTYPES REPORTED IN THE ASYN PFF MODEL}

The earliest aSyn PFF model studies were performed by injecting aSyn PFFs into the mouse striatum. Table 1 provides a summary of studies that used unilateral or bilateral intrastriatal injection of 
Table 1

Injection of mouse aSyn PFFs into the wildtype mouse striatum

\begin{tabular}{|c|c|c|c|c|c|c|c|c|c|c|c|}
\hline \multirow[b]{2}{*}{ Paper } & \multicolumn{8}{|c|}{ Unilateral Mouse aSyn PFFs } & \multicolumn{3}{|c|}{ BilateralMouse aSyn PFFs } \\
\hline & $\begin{array}{l}\text { Luk 2012 } \\
\text { [27] }\end{array}$ & $\begin{array}{c}\text { Masuda-Suzukake } \\
2014 \\
{[28]}\end{array}$ & $\begin{array}{l}\text { Luk 2016 } \\
\text { [19] }\end{array}$ & $\begin{array}{l}\text { Fares 2016 } \\
\text { [29] }\end{array}$ & $\begin{array}{c}\text { Henderson } 2019 \\
{[30]}\end{array}$ & $\begin{array}{l}\text { Izco } 2020 \\
\text { [31] }\end{array}$ & $\begin{array}{l}\text { Burtscher } 2020 \\
\text { [32] }\end{array}$ & $\underset{[33]}{\text { Kim } 2020}$ & $\begin{array}{c}\text { Sorrentino } 2017 \\
{[34]}\end{array}$ & $\begin{array}{c}\text { Stoyka } 2020 \\
{[35]}\end{array}$ & $\begin{array}{l}\text { Ding } 2021 \\
{[36]}\end{array}$ \\
\hline Rodent Strain & C57B16/C3H & C57B/6J J & C57B16/C3H & C57Bl6/C3H & C57BV6J & C57BL6/C3H & C57BL/6JRj & C57BV/6 & Unspecified & C57B//6J J & C57BL6J \\
\hline Injectate & Mouse aSyn & Mouse aSyn & Mouse aSyn & Mouse aSyn & Mouse aSyn & Mouse aSyn & Mouse aSyn & Mouse aSyn & Mouse aSyn & Mouse aSyn & Mouse aSyn \\
\hline Total Dose & $\begin{array}{c}5 \mathrm{ug} \\
\text { (Unilateral) }\end{array}$ & $\begin{array}{c}10 \text { ug } \\
\text { (Unilateral) }\end{array}$ & $\begin{array}{c}5 \mathrm{ug} \\
\text { (Unilateral) }\end{array}$ & $\begin{array}{c}5 \mathrm{ug} \\
\text { (Unilateral) }\end{array}$ & $\begin{array}{c}5 \mathrm{ug} \\
\text { (Unilateral) }\end{array}$ & $\begin{array}{c}5 \mathrm{ug} \\
\text { (Unilateral) }\end{array}$ & $\begin{array}{c}5 \mathrm{ug} \\
\text { (Unilateral) }\end{array}$ & $\begin{array}{c}5 \mathrm{ug} \\
\text { (Unilateral) }\end{array}$ & $\begin{array}{c}4 \text { ug } \\
\text { (Bilateral) }\end{array}$ & $\begin{array}{l}\text { 2ul of 300uM } \\
\text { (Bilateral) }\end{array}$ & $\begin{array}{l}\text { Unspecified } \\
\text { (Bilateral) }\end{array}$ \\
\hline DPI & $30,90,180$ & 30,90 & $14,30,90,180$ & 30 & $30,90,180$ & $15,30,90$ & $30,60,180$ & 30 & 120 & 90,180 & $30,90,180,270$ \\
\hline pS129 aSyn & $30+$ & 30 & $14+$ & 30 & $30+$ & $15+$ & $30+$ & 30 & 120 & $90+$ & $30+$ \\
\hline $\begin{array}{l}\text { Striatal TH } \\
\text { Loss / DA } \\
\text { Deficits }\end{array}$ & $90+$ & N/A & N/A & N/A & N/A & 90 & N/A & 30 & N/A & N/A & N/A \\
\hline $\begin{array}{c}\text { SNpc TH+Cell } \\
\text { Loss }\end{array}$ & $90+$ & N/A & 180 & N/A & $90+$ & Absent & N/A & N/A & Absent & N/A & N/A \\
\hline $\begin{array}{c}\text { Behavioral } \\
\text { Deficits }\end{array}$ & Motor - 180 & $\begin{array}{c}\text {-Motor - } 90 \\
\cdot \text {-Cognitive - Absent }\end{array}$ & Motor - 180 & N/A & $90+$ & Motor - 90 & N/A & Absent & N/A & $\begin{array}{l}\text {-Motor - Absent } \\
\text {-Anxiety - Absent } \\
\text {-Memory - } 180 \\
\text {-Social - } 180\end{array}$ & $\begin{array}{l}\text { •Motor - } 180 \\
\text {-Memory - } 180\end{array}$ \\
\hline $\begin{array}{l}\text { Immune } \\
\text { Response }\end{array}$ & N/A & N/A & N/A & N/A & N/A & $\begin{array}{l}\text {-Microglia Activation } \\
\text { (SNpc) - } 15,90 \\
\text {-Astrogliosis (SNpc, } \\
\text { STR) - } 15+ \\
\text {-Cytokines (SNpc, } \\
\text { STR) - 15, } 90\end{array}$ & N/A & $\begin{array}{l}\text {-Microglia Activation - } \\
\text { Absent } \\
\text {-Astrogliosis - Absent }\end{array}$ & $\begin{array}{l}\text {-Microglia Activation - } \\
\text { Absent } \\
\text {-Astrogliosis - Absent }\end{array}$ & N/A & $\begin{array}{c}\cdot \text { Macrophages with } \\
\text { pS129 aSyn } \\
\text { (Meninges) - } 180+ \\
\text { Cyytokines } \\
\text { (Meninges) - } 180+\end{array}$ \\
\hline Other & N/A & $\begin{array}{c}\text {-Aggregated aSyn - } \\
90 \\
\text {-Tau Pathology - } 30 \\
\text {-TDP-43 Pathology - } \\
30\end{array}$ & Aggregated aSyn - 90 & $\begin{array}{l}\text { Ubiquitinated } \\
\text { Inclusions - } 30\end{array}$ & N/A & N/A & $\begin{array}{c}\text {-TOMM20 in } \\
\text { Inclusions (AMY) - } 60 \\
\text {-VDAC1 Change - } \\
\text { Absent } \\
\text { •Mitochondrial } \\
\text { Respirometry Deficits } \\
\text { - Absent } \\
\text {-ROS - Absent }\end{array}$ & 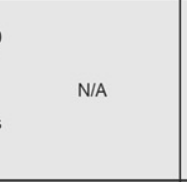 & N/A & $\begin{array}{l}\text { Cell Loss (AMY) - } \\
\text { Absent }\end{array}$ & $\begin{array}{c}\text {-Aggregated aSyn - } \\
180 \\
\cdot \text { Decreased } \\
\text { Meningeal Lymphatic } \\
\text { Drainage }-180+ \\
\text {-Loss of Meningeal } \\
\text { Tight Junctions - } \\
180+\end{array}$ \\
\hline Note & $\begin{array}{l}\mathrm{CD} 1 \text { and BI6/SJL } \\
\text { rodent strains also } \\
\text { assessed }\end{array}$ & & & $\begin{array}{l}\text { Injected human asyn } \\
\text { PFFs and saw little } \\
\text { pathology. }\end{array}$ & $\begin{array}{c}\text { Analyzes spread } \\
\text { patterns and regional } \\
\text { vulnerabilities }\end{array}$ & & & & $\begin{array}{l}\text { Pathology absent with } \\
\text { injection of human } \\
\text { aSyn PFFs }\end{array}$ & & $\begin{array}{l}\text { Pathology worse } \\
\text { when meningeal } \\
\text { lymphatic drainage } \\
\text { blocked }\end{array}$ \\
\hline
\end{tabular}

aSyn, alpha-synuclein; PFFs, preformed fibrils; TH, tyrosine hydroxylase; DA, dopamine; N/A, not analyzed; SNpc, substantia nigra pars compacta; STR, striatum; AMY, amygdala; ROS, reactive oxygen species. 
Table 2

Unilateral injection of human aSyn PFFs into the wildtype mouse striatum

\begin{tabular}{|c|c|c|c|}
\hline & & Human aSyn PFFs & \\
\hline Paper & $\begin{array}{l}\text { Luk 2016 } \\
{[19]}\end{array}$ & $\begin{array}{c}\text { Fares 2016 } \\
\text { [29] }\end{array}$ & $\begin{array}{c}\text { Milanese } 2018 \\
{[37]}\end{array}$ \\
\hline Rodent Strain & C57Bl6/C3H & C57BI6/C3H & C57BI/6 \\
\hline Injectate & Human aSyn & Human aSyn & Human aSyn \\
\hline Total Dose & $\begin{array}{c}5 \mathrm{ug} \\
\text { (Unilateral) }\end{array}$ & $\begin{array}{c}5 \mathrm{ug} \\
\text { (Unilateral) }\end{array}$ & $\begin{array}{c}5 \mathrm{ug} \\
\text { (Unilateral) }\end{array}$ \\
\hline DPI & $14,30,90,180$ & 30 & 120 \\
\hline pS129 aSyn & $30+$ & 30 & 120 \\
\hline $\begin{array}{l}\text { Striatal TH Loss I } \\
\text { DA Deficits }\end{array}$ & N/A & N/A & 120 \\
\hline SNpc TH+ Cell Loss & Absent & N/A & 120 \\
\hline Behavioral Deficits & Absent & N/A & N/A \\
\hline Immune Response & N/A & N/A & N/A \\
\hline Other & Aggregated aSyn - 90 & N/A & DNA Damage (SN) - 120 \\
\hline Note & $\begin{array}{c}\text { Also tested chimeric human-mouse } \\
\text { aSyn PFFs. Homology to mouse } \\
\text { aSyn increased pathology }\end{array}$ & $\begin{array}{l}\text { Injected mouse aSyn PFFs. } \\
\text { Pathology with mouse aSyn PFFs } \\
\text { greater than human aSyn PFFs. }\end{array}$ & \\
\hline
\end{tabular}

aSyn, alpha-synuclein; PFFs, preformed fibrils; TH, tyrosine hydroxylase; DA, dopamine; N/A, not analyzed; SNpc, substantia nigra pars compact.

Table 3

Unilateral and bilateral injection of aSyn PFFs into transgenic mouse striatum

\begin{tabular}{|c|c|c|c|c|c|c|c|c|}
\hline \multirow[b]{2}{*}{ Paper } & \multicolumn{4}{|c|}{ Synuclein Overexpression } & \multicolumn{4}{|c|}{ Other } \\
\hline & $\begin{array}{l}\text { Luk 2012 } \\
\text { [23] }\end{array}$ & \multicolumn{2}{|c|}{$\begin{array}{c}\text { Sorrentino } 2017 \\
{[34]}\end{array}$} & $\begin{array}{c}\text { Earls } 2020 \\
\text { [38] }\end{array}$ & $\begin{array}{c}\text { Blumenstock } 2017 \\
\text { [39] }\end{array}$ & $\begin{array}{c}\text { Henderson } 2019 \\
{[30]}\end{array}$ & $\begin{array}{l}\text { Bieri } 2019 \\
{[40]}\end{array}$ & $\begin{array}{l}\text { Migdalska- } \\
\text { Richards } 2020 \text { [41] }\end{array}$ \\
\hline $\begin{array}{l}\text { Rodent } \\
\text { Strain }\end{array}$ & M83 A53T Hu aSyn & \multicolumn{2}{|c|}{ M20 WT Hu aSyn } & M83 A53T Hu aSyn & Thy-1 eGFP & BAC LRRK2 G2019S & BAC LRRK2 G2019S & GBA L444P KI \\
\hline Injectate & Human aSyn & Human aSyn & Mouse aSyn & Human aSyn & Mouse aSyn & Mouse aSyn & Mouse aSyn & Mouse aSyn \\
\hline Total Dose & $\begin{array}{c}5 \text { ug } \\
\text { (Unilateral) }\end{array}$ & $\begin{array}{c}4 \text { ug } \\
\text { (Bilateral) }\end{array}$ & $\begin{array}{c}4 \text { ug } \\
\text { (Bilateral) }\end{array}$ & $\begin{array}{c}5 \text { ug } \\
\text { (Unilateral) }\end{array}$ & $\begin{array}{l}25 \mathrm{ug} \\
\text { (Unilateral) }\end{array}$ & $\begin{array}{c}5 \mathrm{ug} \\
\text { (Unilateral) }\end{array}$ & $\begin{array}{c}5 \text { ug } \\
\text { (Unilateral) }\end{array}$ & $\begin{array}{c}5 \mathrm{ug} \\
\text { (Unilateral) }\end{array}$ \\
\hline DPI & 90 & 120 & 120 & 70 & $30,60,90,150,270$ & $30,90,180$ & $30,90,180$ & 120 \\
\hline pS129 aSyn & 90 & 120 & 120 & 70 & $30+$ & $30+$ & $30+$ & 120 \\
\hline $\begin{array}{c}\text { Striatal TH } \\
\text { Loss / DA } \\
\text { Deficits }\end{array}$ & $\mathrm{N} / \mathrm{A}$ & N/A & N/A & Absent & N/A & N/A & N/A & $\mathrm{N} / \mathrm{A}$ \\
\hline $\begin{array}{c}\text { SNpc DA Cell } \\
\text { Loss }\end{array}$ & $\mathrm{N} / \mathrm{A}$ & 120 & 120 & Absent & N/A & $90+$ & 180 & N/A \\
\hline $\begin{array}{c}\text { Behavioral } \\
\text { Deficits }\end{array}$ & N/A & N/A & N/A & Motor - 70 & N/A & Motor - $90+$ & Motor - 180 & N/A \\
\hline $\begin{array}{l}\text { Immune } \\
\text { Response }\end{array}$ & $\mathrm{N} / \mathrm{A}$ & $\begin{array}{c}\text {-Microglia Activation } \\
\text { (CPu injection) - } 120 \\
\text { •Astrogliosis (CPu } \\
\text { injection) - } 120 \\
\text { •pS129 aSyn in } \\
\text { Astrocytes - } 120\end{array}$ & $\begin{array}{l}\text {-Microglia Activation - } \\
\text { Absent } \\
\text {-Astrogliosis - Absent }\end{array}$ & $\begin{array}{c}\text {-Microglia Activation - } \\
70 \\
\text {-NK cell infiltration } \\
(\mathrm{SNpc})-70\end{array}$ & $\begin{array}{c}\text { Microglial Activation - } \\
150 \text { (variable) }\end{array}$ & N/A & $\begin{array}{c}\text { Microglia Activation - } \\
180\end{array}$ & $\mathrm{~N} / \mathrm{A}$ \\
\hline Other & $\mathrm{N} / \mathrm{A}$ & N/A & N/A & Aggregated aSyn - 70 & $\begin{array}{l}\text {-Ubiquitin and Thios } \\
\text { Inclusions - } 150 \\
\cdot \text { Dendrite } \\
\text { Malformations (CTX) - } \\
150 \\
\text {-Dendritic Spine Loss } \\
\text { (CTX) - } 150\end{array}$ & N/A & & N/A \\
\hline Note & $\begin{array}{l}\text { Also injected truncated } \\
\text { (AA1-120) human aSyn } \\
\text { PFFs }\end{array}$ & $\begin{array}{c}\text { Tested different } \\
\text { injection coordinates }\end{array}$ & & $\begin{array}{c}\text { NK cell depletion } \\
\text { worsened pathology }\end{array}$ & & $\begin{array}{c}\text { Pathology in LRRK2 } \\
\text { G2019S mouse worse } \\
\text { than WT }\end{array}$ & $\begin{array}{c}\text { Pathology in LRRK2 } \\
\text { G2019S mouse worse } \\
\text { than WT }\end{array}$ & $\begin{array}{l}\text { Pathology in GBA } \\
\text { L444P mouse worse } \\
\text { than WT }\end{array}$ \\
\hline
\end{tabular}

aSyn, alpha-synuclein; PFFs, preformed fibrils; Hu, human; TH, tyrosine hydroxylase; DA, dopamine; N/A, not analyzed; SNpc, substantia nigra pars compacta; $\mathrm{CPu}$, caudate putamen; $\mathrm{CTX}$, cortex. 
Table 4

Unilateral and bilateral injection of aSyn PFFs into the wildtype and transgenic mouse olfactory bulb or sublaterodorsal tegmental nucleus

\begin{tabular}{|c|c|c|c|c|c|c|c|c|c|}
\hline \multirow{3}{*}{$\begin{array}{l}\text { Paper } \\
\text { Rodent } \\
\text { Strain }\end{array}$} & \multicolumn{8}{|c|}{ Olfactory Bulb } & \multirow{3}{*}{\begin{tabular}{|c}
$\begin{array}{c}\text { Sublaterodorsal } \\
\text { Tegmental } \\
\text { Nucleus (SLD) }\end{array}$ \\
$\begin{array}{c}\text { Shen 2020 } \\
\text { [47] }\end{array}$ \\
C57BV/6
\end{tabular}} \\
\hline & \multicolumn{2}{|c|}{$\begin{array}{l}\text { Rey } 2016 \\
\text { [42] }\end{array}$} & \multicolumn{2}{|c|}{$\begin{array}{c}\text { Rey } 2018 \\
\text { [43] }\end{array}$} & \multirow{2}{*}{$\begin{array}{c}\text { Graham } 2018 \\
\text { [44] } \\
\text { C57BV/6 }\end{array}$} & \multirow{2}{*}{$\begin{array}{c}\text { Kulkarni } 2020 \\
\text { [45] } \\
\text { C57BL/6J }\end{array}$} & \multicolumn{2}{|c|}{$\begin{array}{c}\text { Uemura } 2021 \\
\text { [46] }\end{array}$} & \\
\hline & C57BV/6 & C57BI/6 & C57BV/6J & C57BI/6J & & & C57BL/6J & $\begin{array}{l}\text { BAC Hu A53T } \\
\text { aSyn Mouse }\end{array}$ & \\
\hline Injectate & Mouse aSyn & Human aSyn & Mouse aSyn & Human aSyn & Human aSyn & Mouse aSyn & Mouse aSyn & Mouse aSyn & $\begin{array}{l}\text { Atto } 488 \text {-labeled } \\
\text { human aSyn }\end{array}$ \\
\hline Total Dose & $\begin{array}{c}4 \text { ug } \\
\text { (Unilateral) }\end{array}$ & $\begin{array}{c}4 \text { ug } \\
\text { (Unilateral) }\end{array}$ & $\begin{array}{c}4 \text { ug } \\
\text { (Unilateral) }\end{array}$ & $\begin{array}{c}4 \text { ug } \\
\text { (Unilateral) }\end{array}$ & $\begin{array}{c}4 \text { ug } \\
\text { (Unilateral) }\end{array}$ & Unclear & $\begin{array}{c}2.5 \mathrm{ug} \\
\text { (Bilateral) }\end{array}$ & $\begin{array}{c}2.5 \mathrm{ug} \\
\text { (Bilateral) }\end{array}$ & Unclear \\
\hline DPI & $30,90,180,360$ & $30,90,180,360$ & $270,540,690$ & $270,540,690$ & 90 & $30,60,90$ & 60 & $\begin{array}{c}60,180,210,240 \\
300\end{array}$ & $\begin{array}{c}30,60,90,150 \\
240\end{array}$ \\
\hline pS129 aSyn & $30+$ & $30+$ & 270 & $270+$ & N/A & $30,60,90$ & 60 & $60+$ & $30+$ \\
\hline Cell Loss & Absent (OB) & Absent (OB) & $\begin{array}{c}180(\mathrm{AON}) \\
\text { Absent (OB) }\end{array}$ & $\begin{array}{c}180(\mathrm{AON}) \\
\text { Absent (OB) }\end{array}$ & $\mathrm{N} / \mathrm{A}$ & $\mathrm{N} / \mathrm{A}$ & N/A & 300 (HPC) & $\begin{array}{c}30+(\mathrm{SLD}) \\
90+(\mathrm{SNpc})\end{array}$ \\
\hline $\begin{array}{l}\text { Behavioral } \\
\text { Deficits }\end{array}$ & $\begin{array}{l}\text {-Motor - Absent } \\
\text {-Anxiety - Absent } \\
\text {-Olfactory - } 30\end{array}$ & $\begin{array}{l}\text {-Motor - Absent } \\
\text {-Anxiety - Absent } \\
\text {-Olfactory - } 30\end{array}$ & $\mathrm{~N} / \mathrm{A}$ & N/A & N/A & N/A & N/A & $\begin{array}{l}\text {-Memory - } 240 \\
\text {-Olfactory - } 60 \\
\text {-Anxiety - } 210\end{array}$ & $\begin{array}{c}\text { •Motor - } 150+ \\
\text {-Olfactory - } 150+ \\
\text { ·GI - } 150+ \\
\text { •RBD - 30+ }\end{array}$ \\
\hline $\begin{array}{l}\text { Immune } \\
\text { Response }\end{array}$ & $\begin{array}{c}\text {-Microglia } \\
\text { Activation - Absent }\end{array}$ & $\begin{array}{c}\text { •Microglia } \\
\text { Activation - Absent }\end{array}$ & N/A & N/A & N/A & N/A & N/A & $\begin{array}{c}\text { •Microglia } \\
\text { Activation - } 180+ \\
\text {-Astrogliosis - } 180+\end{array}$ & N/A \\
\hline Other & $\begin{array}{c}\text {-Aggregated aSyn } \\
-30+ \\
\text {-Tau pathology - } \\
\text { Absent }\end{array}$ & $\begin{array}{c}\text {-Aggregated aSyn } \\
-30+ \\
\text {-Tau pathology - } \\
\text { Absent }\end{array}$ & $\begin{array}{c}\text {-Tau pathology - } \\
\text { Absent } \\
\text {-TDP-43 pathology } \\
\text { - Absent }\end{array}$ & $\begin{array}{c}\text {-Tau pathology - } \\
\text { Absent } \\
\text { •TDP-43 pathology } \\
\text { - Absent }\end{array}$ & $\begin{array}{l}\text { Phospholipid } \\
\text { alterations (brain, } \\
\text { serum) - } 90\end{array}$ & $\begin{array}{l}\text {-Spontaneous LFP } \\
\text { activity changes - } \\
\text { absent } \\
\text { - Odor-evoked beta } \\
\text { band increase - } \\
30+ \\
\end{array}$ & $\begin{array}{c}\text { Aggregated aSyn } \\
60\end{array}$ & $\begin{array}{c}- \text { Aggregated aSyn - } \\
60+\end{array}$ & $\begin{array}{c}\text {-Striatal TH+ fiber } \\
\text { loss - } 30+ \\
\text {-Striatal DA deficits } \\
-90+\end{array}$ \\
\hline Note & $\begin{array}{l}\text { Mouse aSyn PF } \\
\text { pathology than } \mathrm{h}\end{array}$ & $\begin{array}{l}\text { Fs induced more } \\
\text { uman aSyn PFFs }\end{array}$ & $\begin{array}{c}\text { pS129 aSyn } \\
\text { pathology } \\
\text { decreased after } 18 \\
\text { months }\end{array}$ & $\begin{array}{l}\text { pS129 aSyn } \\
\text { pathology } \\
\text { remained static } \\
\text { after } 12 \text { months }\end{array}$ & $\begin{array}{l}\text { Pooled injected \& } \\
\text { uninjected } \\
\text { hemisphere and all } \\
\text { brain structures }\end{array}$ & & & & \\
\hline
\end{tabular}

aSyn, alpha-synuclein; PFFs, preformed fibrils; Hu, human; N/A - not analyzed; OB, olfactory bulb; AON, accessory olfactory nucleus; HPC, hippocampus; SLD, subdorsolateral tegmental nucleus; SNpc, substantia nigra pars compacta; GI, gastrointestinal; RBD, REM sleep behavior disorder; LFP, local field potential; TH, tyrosine hydroxylase; DA, dopamine.

Table 5

Unilateral or bilateral injection of aSyn PFFs into the wildtype or transgenic mouse hippocampus, cortex, or substantia nigra

\begin{tabular}{|c|c|c|c|c|c|c|c|c|c|}
\hline & \multicolumn{4}{|c|}{ Hippocampus } & \multicolumn{3}{|c|}{ Cortex } & \multicolumn{2}{|c|}{ Substantia Nigra } \\
\hline Paper & $\begin{array}{l}\text { Rutherford } 2017 \\
\text { [20] }\end{array}$ & $\begin{array}{l}\text { Luna } 2018 \\
{[48]}\end{array}$ & $\begin{array}{l}\text { Nouraei } 2019 \\
{[49]}\end{array}$ & $\begin{array}{c}\text { Caputo } 2020 \\
{[50]}\end{array}$ & $\begin{array}{c}\text { Masuda- } \\
\text { Suzukake 2014 } \\
\text { [28] }\end{array}$ & $\begin{array}{l}\text { Osterberg } 2015 \\
\text { [51] }\end{array}$ & $\begin{array}{c}\text { Schaser } 2020 \\
{[52]}\end{array}$ & $\begin{array}{l}\text { Masuda- } \\
\text { Suzukake 2013 } \\
\text { [53] }\end{array}$ & $\begin{array}{c}\text { Masuda- } \\
\text { Suzukake 2014 } \\
\text { [28] }\end{array}$ \\
\hline $\begin{array}{l}\text { Rodent } \\
\text { Strain }\end{array}$ & M20 WT Hu aSyn & C57B16/C3H & CD1 & $\begin{array}{c}\text { GFP-tagged SNCA } \\
\mathrm{KI}\end{array}$ & C57BV6J & $\begin{array}{l}\text { GFP-tagged Hu } \\
\text { SNCA }\end{array}$ & $\begin{array}{l}\text { GFP-tagged Hu } \\
\text { A53T SNCA }\end{array}$ & C57B//6J J & C57B/6J J \\
\hline Injectate & Human aSyn & Mouse aSyn & Mouse aSyn & Mouse aSyn & Mouse aSyn & Mouse aSyn & Mouse aSyn & Human aSyn & Mouse aSyn \\
\hline Total Dose & $\begin{array}{c}4 \mathrm{ug} \\
\text { (Bilateral) }\end{array}$ & $\begin{array}{l}5 \mathrm{ug} \\
\text { (Unilateral) }\end{array}$ & $\begin{array}{l}5 \mathrm{ug} \\
\text { (Unilateral) }\end{array}$ & $\begin{array}{l}5 \mathrm{ug} \\
\text { (Unilateral) }\end{array}$ & $\begin{array}{l}10 \mathrm{ug} \\
\text { (Unilateral) }\end{array}$ & $\begin{array}{l}5 \mathrm{ug} \\
\text { (Unilateral) }\end{array}$ & $\begin{array}{l}5 \mathrm{ug} \\
\text { (Unilateral) }\end{array}$ & $\begin{array}{l}10 \mathrm{ug} \\
\text { (Unilateral) }\end{array}$ & $\begin{array}{c}10 \mathrm{ug} \\
\text { (Unilateral) }\end{array}$ \\
\hline DPI & 60,120 & 45,90 & 90 & 30 & 30,90 & $30.90,210$ & $10,20,30,50$ & $90,180,450$ & 30,90 \\
\hline pS129 aSyn & $60+$ & $45+$ & 90 & 30 & 30 & $30+$ & $10+$ & $90+$ & 30 \\
\hline Cell Loss & N/A & 45,90 & Absent (HPC) & N/A & N/A & 210 & $30+(\mathrm{CTX})$ & Absent (SN) & N/A \\
\hline \begin{tabular}{|c|} 
Behavioral \\
Deficits
\end{tabular} & N/A & N/A & $\begin{array}{l}\text {-Memory - Absent } \\
\text {-Olfactory - Absent }\end{array}$ & N/A & \begin{tabular}{|l} 
- Motor - Absent \\
$\cdot$ Cognitive - Absent
\end{tabular} & N/A & N/A & Motor - Absent & $\begin{array}{c}\text {-Motor - } 90 \\
\text {.Cognitive - Absent }\end{array}$ \\
\hline \begin{tabular}{|c|} 
Immune \\
Response
\end{tabular} & N/A & N/A & N/A & N/A & N/A & N/A & N/A & $\begin{array}{l}\text {-Microglial Activation } \\
\text {-Absent } \\
\text {-Astrogliosis - } \\
\text { Absent }\end{array}$ & N/A \\
\hline Other & N/A & N/A & $\begin{array}{c}\text {-Synaptophysin } \\
\text { increase (CA2/CA3) } \\
-90 \\
\text { - Ubiquitin and ThioS } \\
\text { inclusions - } 90\end{array}$ & N/A & $\begin{array}{c}\text {-Tau pathology - } 30 \\
\text {-TDP-43 pathology - } \\
90 \\
\text {-Aggregated aSyn - } \\
90\end{array}$ & $\begin{array}{l}\text { Ubiquitinated } \\
\text { inclusions - } 90\end{array}$ & $\begin{array}{l}\text { PS129 aSyn in } \\
\text { GFAP+ cells - 50+ }\end{array}$ & $\begin{array}{l}\text {-Aggregated aSyn - } \\
90 \\
\text {-Ubiquitin and p62 } \\
\text { inclusions - } 450\end{array}$ & $\begin{array}{c}\text {-Tau pathology - } \\
\text { Absent } \\
\text {-TDP-43 pathology - } \\
\text { Absent } \\
\text {-Aggregated aSyn - } \\
90\end{array}$ \\
\hline Note & $\begin{array}{l}\text { Also used mutant } \\
\text { aSyn PFFs }\end{array}$ & $\begin{array}{c}\text { MATH2+ and } \\
\text { CTIP2+ cells more } \\
\text { susceptible than } \\
\text { Prox } 1+\end{array}$ & & $\begin{array}{c}\text { pS129 aSyn } \\
\text { pathology reduced } \\
\text { with GFP dosing }\end{array}$ & & $\begin{array}{l}\text { Performed in vivo } \\
\text { imaging for } \\
\text { longitudinal } \\
\text { assessments }\end{array}$ & $\begin{array}{l}\text { Performed in vivo } \\
\text { imaging for } \\
\text { longitudinal } \\
\text { assessments }\end{array}$ & \begin{tabular}{|c|} 
Injection of mouse \\
aSyn PFFs resulted \\
in similar pS129 \\
aSyn
\end{tabular} & \\
\hline
\end{tabular}

aSyn, alpha-synuclein; PFFs, preformed fibrils; Hu, human; N/A, not analyzed; KI, knockin; HPC, hippocampus; CTX, cortex; SN, substantia nigra.

mouse aSyn PFFs in wildtype (WT) mice. Table 2 provides a summary of studies that used intrastriatal injection of human aSyn PFFs in WT mice. Table 3 provides a summary of studies that used intrastriatal injection in transgenic mice.
Others have chosen to inject non-striatal brain regions to model prodromal or non-motor features of PD in mice. Table 4 provides a summary of studies injecting aSyn PFFs into the olfactory bulb (OB) or sublaterodorsal tegmental nucleus (SLD) to 
Table 6

Unilateral or bilateral injection of aSyn PFFs into the wildtype or knockout rat striatum or substantia nigra

\begin{tabular}{|c|c|c|c|c|c|c|c|c|}
\hline \multirow{3}{*}{$\begin{array}{l}\text { Paper } \\
\text { Rodent } \\
\text { Strain }\end{array}$} & \multicolumn{6}{|c|}{ Striatum } & \multicolumn{2}{|c|}{$\overline{S N p c}$} \\
\hline & \multirow{2}{*}{$\begin{array}{c}\begin{array}{c}\text { Paumier } 2015 \\
\text { [54] }\end{array} \\
\text { Sprague Dawley }\end{array}$} & \multirow{2}{*}{$\begin{array}{c}\begin{array}{c}\text { Duffy } 2018 \\
\text { [55] }\end{array} \\
\text { Fischer } 344\end{array}$} & \multicolumn{2}{|c|}{$\begin{array}{c}\text { Patterson } 2019 \\
{[56]}\end{array}$} & \multirow{2}{*}{$\begin{array}{l}\begin{array}{c}\text { Thomsen } 2021 \\
\text { [57] }\end{array} \\
\text { Sprague Dawley }\end{array}$} & \multirow{2}{*}{$\begin{array}{c}\text { Creed 2020 } \\
\text { [58] } \\
\text { WT and PINK1 KO } \\
\text { Rat }\end{array}$} & \multirow{2}{*}{$\begin{array}{c}\text { Thakur } 2017 \\
\text { [59] } \\
\text { Sprague Dawley }\end{array}$} & \multirow{2}{*}{$\begin{array}{c}\begin{array}{c}\text { Harms } 2017 \\
{[60]}\end{array} \\
\text { Sprague Dawley }\end{array}$} \\
\hline & & & Fischer 344 & Fischer 344 & & & & \\
\hline Injectate & Mouse aSyn & Mouse aSyn & Mouse aSyn & Mouse aSyn & Mouse aSyn & Mouse aSyn & Human aSyn & Mouse aSyn \\
\hline Total Dose & $\begin{array}{c}8 \mathrm{ug} \\
\text { (Unilateral) }\end{array}$ & $\begin{array}{c}\text { 8ug } \\
\text { (Unilateral) }\end{array}$ & $\begin{array}{l}\text { 8ug or } 16 \text { ug } \\
\text { (Unilateral) }\end{array}$ & $\begin{array}{c}16 \text { ug } \\
\text { (Bilateral) }\end{array}$ & $\begin{array}{c}8 \mathrm{ug} \\
\text { (Unilateral) }\end{array}$ & $\begin{array}{c}20 \text { ug } \\
\text { (Unilateral) }\end{array}$ & Unclear & $\begin{array}{c}8 \mathrm{ug} \\
\text { (Unilateral) }\end{array}$ \\
\hline DPI & $30,60,90,180$ & $\begin{array}{c}14,30,60,90,120 \\
150,180\end{array}$ & $60,120,180$ & 180 & $45,60,100,120$ & 14,30 & $10,20,90,180$ & $30,60,90,180$ \\
\hline ps129 aSyn & $30+$ & $30+$ & $60+$ & 180 & $45+$ & $14+$ & $10+$ & $\begin{array}{c}30+(\mathrm{SN}) \\
60+(\mathrm{STR})\end{array}$ \\
\hline $\begin{array}{l}\text { Striatal TH } \\
\text { Loss / DA } \\
\text { Deficits }\end{array}$ & $\begin{array}{c}\text {-Neurochemistry } \\
\text { deficits }-180 \\
\text {-TH increase }-30 \\
\text {-TH decrease }-60+\end{array}$ & N/A & TH decrease - $60+$ & N/A & $\begin{array}{c}\text {-TH decrease - 45+ } \\
\text {-VMAT decrease - } \\
45+ \\
\text {-DAT decrease - } \\
100+\end{array}$ & Absent & TH decrease - $90+$ & TH decrease - $90+$ \\
\hline $\begin{array}{l}\text { SNpc DA } \\
\text { Cell Loss }\end{array}$ & 180 & $150+$ & $\begin{array}{c}120+\text { (16ug) } \\
180 \text { (8ug) }\end{array}$ & 180 & $100+$ & $\begin{array}{l}\text { WT - Absent } \\
\text { PINK1 KO - 30 }\end{array}$ & $20+$ & $90+$ \\
\hline $\begin{array}{l}\text { Behavioral } \\
\text { Deficits }\end{array}$ & $\begin{array}{c}\cdot \text { Motor - Absent } \\
\cdot \text {-Vocalizations }-180\end{array}$ & N/A & $\begin{array}{c}\text {-Motor (8ug) - N/A } \\
\text {-Motor (16ug) - } \\
\text { Absent }\end{array}$ & Motor - 180 & $\begin{array}{c}\text { Sensorimotor - } 60 \\
\text { (absent 120) }\end{array}$ & N/A & Motor - Absent & N/A \\
\hline $\begin{array}{l}\text { Immune } \\
\text { Response }\end{array}$ & $\mathrm{N} / \mathrm{A}$ & $\begin{array}{c}\text { Microglia Activation - } \\
60+\end{array}$ & N/A & N/A & $\begin{array}{c}\cdot \text { Microglia Activation } \\
(\text { SN) - } 45,100 \\
\text {-Microglia Activation } \\
\text { (STR) - } 100\end{array}$ & N/A & $\begin{array}{l}\text { Microgliosis - } 10 \\
\text { (absent 90, 180) }\end{array}$ & $\begin{array}{l}\text {-Microglia Activation } \\
\text { (SN) - 30+ } \\
\text {-Microglia Activation } \\
\text { (STR) - } 180 \\
\text {-Monocyte infiltration } \\
\text { (SN, STR) }-60 \\
\text {-T cell infiltration } \\
\text { (SN) - } 60\end{array}$ \\
\hline Other & & $\begin{array}{l}\text { aSyn Aggregates - } \\
\qquad 30+\end{array}$ & $\begin{array}{l}\text { aSyn Aggregates - } \\
30+\end{array}$ & N/A & $\begin{array}{l}\text { aSyn Aggregates - } \\
45+\end{array}$ & aSyn Aggregates - & N/A & N/A \\
\hline Note & $\begin{array}{c}\text { TH phenotype in } \\
\text { SNpc downregulated } \\
\text { before neuronal loss }\end{array}$ & $\begin{array}{l}\text { Reactive microglia } \\
\text { associated with } \\
\text { pS129 aSyn but } \\
\text { reduces during } \\
\text { degeneration }\end{array}$ & $\begin{array}{r}\text { More pathology } \\
\text { Pathology more seve } \\
\text { injec }\end{array}$ & $\begin{array}{l}16 \text { ug vs } 8 \text { ug. } \\
\text { with bilateral PFF } \\
\text { n. }\end{array}$ & & $\begin{array}{l}\text { Pathology in PINK1 } \\
\text { KO worse than WT }\end{array}$ & $\begin{array}{c}\text { TH phenotype in } \\
\text { SNpc downregulated } \\
\text { pefore neuronal loss; } \\
\text { Pathology worse } \\
\text { with AAV aSyn } \\
\text { addition }\end{array}$ & \\
\hline
\end{tabular}

aSyn, alpha-synuclein; PFFs, preformed fibrils; SNpc, substantia nigra pars compacta; WT, wildtype; KO, knockout; TH, tyrosine hydroxylase; DA, dopamine; VMAT, vesicular monoamine transporter; DAT, dopamine transporter; STR, striatum.

model olfactory dysfunction and sleep disturbances, respectively. Table 5 provides a summary of studies injecting aSyn PFFs into the hippocampus, cortex, and $\mathrm{SNpc}$ as alternate ways to induce pathology in the mouse.

Although most studies to date have focused on phenotyping mice injected with aSyn PFFs, rats have also been used for this model. Table 6 provides a summary of studies injecting aSyn PFFs into the rat striatum or SNpc.

In addition, both mice and rats have been used for peripheral administration of aSyn PFFs to study the seeding capabilities of aSyn PFFs and peripheralto-central spread of synuclein pathology. Table 7 provides a summary of studies injecting aSyn PFFs into the gut of rodents to model GI dysfunction and gut-to-brain transmissibility of aSyn pathology. Finally, Table 8 provides a summary of studies performing intramuscular injections of aSyn PFFs into rodents and Table 9 provides a summary of studies performing intraperitoneal, intravenous, intraneural, and intravitreal injection of aSyn PFFs into rodents.
A visual representation of timelines of phenotypes reported in common iterations of the aSyn PFF model is provided in Fig. 1. Replicated phenotypes that have been reported in more than one study are provided along the timeline of the model. Phenotypes that were only investigated in one study are also included but denoted as "underexplored phenotypes". An inset containing phenotypes that were reported as absent is also included.

\section{DISCUSSION}

For all studies, one of the earliest phenotypes reported is the presence of pS129 aSyn within brain regions innervating the injected structure. As the model progresses, the density of pS129 aSyn pathology and regions displaying pS129 aSyn pathology increase. This pathology is at times accompanied by cell loss, inflammation, behavioral deficits, and/or other readouts of pathology. Importantly, the phenotypes observed in this model are not always reproducible and their presence/absence varies between studies (Fig. 1). This can be noted 
Table 7

Injection of aSyn PFFs into the wildtype or transgenic rodent gut

\begin{tabular}{|c|c|c|c|c|c|c|c|c|c|c|c|}
\hline \multirow{3}{*}{$\begin{array}{l}\text { Paper } \\
\text { Rodent } \\
\text { Strain }\end{array}$} & \multicolumn{6}{|c|}{ Mouse } & \multicolumn{5}{|c|}{ Rat } \\
\hline & \multirow{2}{*}{$\begin{array}{c}\text { Uemura } 2018 \\
{[61]} \\
\text { C57BI/J }\end{array}$} & \multirow{2}{*}{$\begin{array}{c}\text { Kim 2019 } \\
\text { [62] } \\
\text { C57BI/J }\end{array}$} & \multirow{2}{*}{$\begin{array}{l}\text { Uemura 2020 } \\
\text { [63] } \\
\begin{array}{c}\text { BAC Hu A53T } \\
\text { aSyn }\end{array}\end{array}$} & \multicolumn{2}{|c|}{$\begin{array}{c}\text { Challis } 2020 \\
{[64]}\end{array}$} & \multirow{2}{*}{$\begin{array}{l}\text { Wang } 2020 \\
\text { [65] } \\
\text { M83 A53T Hu } \\
\text { aSyn }\end{array}$} & \multirow{2}{*}{\begin{tabular}{|c}
$\begin{array}{c}\text { Holmqvist } 2014 \\
\text { [66] }\end{array}$ \\
Sprague Dawley
\end{tabular}} & \multirow{2}{*}{$\begin{array}{c}\text { Manfredsson } \\
2018 \\
{[67]} \\
\text { Sprague Dawley }\end{array}$} & \multicolumn{2}{|c|}{$\begin{array}{c}\text { Van Den Berge } 2019 \\
\text { [68] }\end{array}$} & \multirow{2}{*}{$\begin{array}{c}\text { Van Den Berge } \\
2021 \\
{[69]} \\
\text { Fischer } 344\end{array}$} \\
\hline & & & & $\begin{array}{l}\text { C57B/N } \\
\text { (2 month) }\end{array}$ & $\begin{array}{c}\mathrm{C} 57 \mathrm{~B} / \mathrm{N} \\
\text { (16 month) }\end{array}$ & & & & Sprague Dawley & BAC Hu WT aSyn & \\
\hline Injectate & Mouse aSyn & Mouse aSyn & Mouse aSyn & Mouse aSyn & Mouse aSyn & Human aSyn & Human aSyn & Mouse aSyn & Human aSyn & Human aSyn & Mouse aSyn \\
\hline $\begin{array}{l}\text { Injection } \\
\text { Site }\end{array}$ & $\begin{array}{c}\text { Gastric Wall } \\
\text { (unclear location) }\end{array}$ & $\begin{array}{l}\text { Pylorus \& } \\
\text { Duodenum } \\
\text { (gastric wall) }\end{array}$ & $\begin{array}{l}\text { Pylorus \& Stomach } \\
\text { (gastric wall) }\end{array}$ & $\begin{array}{l}\text { Duodenum } \\
\text { (gastric wall) }\end{array}$ & $\begin{array}{l}\text { Duodenum } \\
\text { (gastric wall) }\end{array}$ & $\begin{array}{l}\text { Stellate ganglia, } \\
\text { Celiac ganglia }\end{array}$ & $\begin{array}{l}\text { Stomach \& } \\
\text { Duodenum } \\
\text { (gastric wall) }\end{array}$ & $\begin{array}{l}\text { Descending Colon } \\
\text { (gastric wall) }\end{array}$ & $\begin{array}{c}\text { Pylorus \& } \\
\text { Duodenum } \\
\text { (gastric wall) }\end{array}$ & $\begin{array}{l}\text { Pylorus \& } \\
\text { Duodenum } \\
\text { (gastric wall) }\end{array}$ & $\begin{array}{c}\text { Pylorus \& } \\
\text { Duodenum } \\
\text { (gastric wall) }\end{array}$ \\
\hline Total Dose & $\begin{array}{c}48 \text { ug } \\
\text { (over } 8 \text { sites) }\end{array}$ & $\begin{array}{c}25 \text { ug } \\
\text { (over } 2 \text { sites) }\end{array}$ & $\begin{array}{c}48 \text { ug } \\
\text { (over } 8 \text { sites) }\end{array}$ & $\begin{array}{c}\text { 6ug } \\
\text { (over } 2 \text { sites) }\end{array}$ & $\begin{array}{c}\text { 6ug } \\
\text { (over } 2 \text { sites) }\end{array}$ & $\begin{array}{c}11 \text { ug } \\
\text { (over } 4 \text { sites) }\end{array}$ & $\begin{array}{c}15 \text { ug } \\
\text { (over } 5 \text { sites) }\end{array}$ & $\begin{array}{c}\text { 60ug } \\
\text { (over } 6 \text { sites) }\end{array}$ & $\begin{array}{l}18 \mathrm{ug} \\
\text { (over } 6 \text { sites) }\end{array}$ & $\begin{array}{c}18 \text { ug } \\
\text { (over } 6 \text { sites) }\end{array}$ & $\begin{array}{c}\text { 60ug } \\
\text { (over } 6 \text { sites) }\end{array}$ \\
\hline DPI & $23,45,180$ & $30,90,210,300$ & $\begin{array}{c}30,60,120,180 \\
240\end{array}$ & $7,21,60,120$ & $7,21,60,120$ & $\begin{array}{c}30,60,90,120, \\
150,180,210 \\
\end{array}$ & $0.5,1,2,3,6$ & $30,180,360$ & 60,120 & 60,120 & 60 \\
\hline $\begin{array}{l}\text { Peripherall } \\
\text { Spinal Cord } \\
\text { pS129 aSyn }\end{array}$ & $\begin{array}{c}45+(\mathrm{DMV}) \\
180(\mathrm{MG})\end{array}$ & $30+(\mathrm{DMV})$ & $\begin{array}{c}\text { 30, } 60 \text { (DMV, MG) } \\
\text { Absent in } \\
\text { duodenum and SC }\end{array}$ & $\begin{array}{c}60 \text { (MG) } \\
\text { Absent (DMV) }\end{array}$ & 120 (DMV) & $\begin{array}{c}30+(\mathrm{SC}) \\
60+(\mathrm{Gl}, \text { skin, } \\
\text { heart, sweat gland) }\end{array}$ & $\begin{array}{c}2+\text { (vagal nerve) } \\
6 \text { (DMV) }\end{array}$ & $\begin{array}{l}30+(\mathrm{MG}) \\
30 \text { (DMV) }\end{array}$ & Absent & $\begin{array}{l}120 \text { (Stomach, } \\
\text { DMV, heart) }\end{array}$ & $\begin{array}{c}60 \text { (MG, DMV, SC, } \\
\text { heart, muscle, } \\
\text { kidney) }\end{array}$ \\
\hline $\begin{array}{c}\text { CNS pS129 } \\
\text { aSyn }\end{array}$ & Absent & $\begin{array}{c}30+(\text { Brainstem }) \\
90+(\text { Midbrain) } \\
210+\text { (Forebrain) }\end{array}$ & $\begin{array}{l}120 \text { (Brainstem) } \\
\text { Absent in Midbrain } \\
\text { and Forebrain }\end{array}$ & Absent (Midbrain) & 120 (Midbrain) & $\begin{array}{l}30+\text { (Brainstem, } \\
\text { Midbrain) }\end{array}$ & Absent & 30 (Brainstem) & Absent & $\begin{array}{l}120 \text { (Brainstem, } \\
\text { Midbrain) }\end{array}$ & $\begin{array}{c}60 \text { (Brainstem, } \\
\text { Midbrain, } \\
\text { Forebrain) }\end{array}$ \\
\hline Cell Loss & N/A & SNpc DA - 210+ & N/A & Absent (MG) & Absent (SN) & N/A & Absent & Absent & N/A & N/A & N.A \\
\hline $\begin{array}{c}\text { Behavioral } \\
\text { Deficits }\end{array}$ & N/A & $\begin{array}{l}\text {-Motor - } 210+ \\
\text { - Cognitive }-210+ \\
\text {-Psychiatric - } 210+ \\
\text {-Olfactory - } 300\end{array}$ & N/A & $\begin{array}{c}\text {-Motor - 60, } 90 \\
\cdot \mathrm{GI}-60+\end{array}$ & $\begin{array}{c}\cdot \text { Motor - } 120 \\
\cdot G I-120\end{array}$ & $\begin{array}{c}\cdot \text { GI }-90+ \\
\text { - Olfactory - } 90+ \\
\text { - Orthostatic } \\
\text { hypotension - } 90+ \\
\cdot \text { Hypohidrosis - } \\
90+\end{array}$ & N/A & N/A & N/A & N/A & N/A \\
\hline Other & $\begin{array}{l}\text { Nitrated aSyn } \\
\text { (DMV) - } 45\end{array}$ & & $\begin{array}{c}\text {-Nitrated aSyn } \\
\text { (DMV, MG) - } 30,60 \\
\text {-Ubiquitin and p62 } \\
\text { (DMV, MG) - } 30,60\end{array}$ & $\begin{array}{c}\text {-Gl inflammation - } \\
7+\text {, } \\
\text {-GCase Decrease } \\
\text { (duodenum) - } 7 \\
\text {-Altered GI network } \\
-7+ \\
\text {-Striatal DA deficits } \\
\text {-Absent }\end{array}$ & $\begin{array}{l}\text { Striatal DA deficits } \\
-120\end{array}$ & Death - 150+ & N/A & N/A & N/A & N/A & N/A \\
\hline Note & $\begin{array}{c}\text { Vagotomy } \\
\text { prevented } \\
\text { pathology in DMV } \\
\text { but reduced } \\
\text { heurons by } 20-40 \%\end{array}$ & $\begin{array}{l}\text { No brain pathology } \\
\text { with vagotomy or } \\
\text { aSyn KO }\end{array}$ & & $\begin{array}{l}\text { AAV-PHP.S } \\
\text { GCase } \\
\text { overexpression } \\
\text { reduced pathology }\end{array}$ & & & & $\begin{array}{l}\text { aSyn pathology was } \\
\text { equal in PFF \& } \\
\text { monomer groups }\end{array}$ & & & $\begin{array}{c}\text { Rats also injected } \\
\text { with human } \\
\text { S129A aSyn } \\
\text { PFFs; pathology } \\
\text { worse with age. } \\
\text { Pathology with } \\
\text { mouse PFFs } \\
\text { greater than } \\
\text { human PFFs. } \\
\end{array}$ \\
\hline
\end{tabular}

aSyn, alpha-synuclein; PFFs, preformed fibrils; Hu, human; DMV, dorsal motor nucleus of the vagus; MG, myenteric ganglia; SC, spinal cord; GI, gastrointestinal system; CNS,

central nervous system; SNpc, substantia nigra pars compacta; DA, dopamine; KO, knockout. 
Table 8

Unilateral or bilateral injection of aSyn PFFs into the transgenic mouse muscle

\begin{tabular}{|c|c|c|c|c|c|c|}
\hline \multirow[b]{2}{*}{ Paper } & \multicolumn{6}{|c|}{ Intramuscular } \\
\hline & $\begin{array}{l}\text { Rutherford } 2017 \\
\text { [20] }\end{array}$ & $\begin{array}{l}\text { Ayers } 2017 \\
{[70]}\end{array}$ & $\begin{array}{c}\text { Sorrentino } 2018 \\
\text { [71] }\end{array}$ & $\begin{array}{c}\text { Schaser } 2020 \\
\text { [52] }\end{array}$ & $\begin{array}{l}\text { Chu 2020 } \\
\text { [72] }\end{array}$ & $\begin{array}{c}\text { Ferreira } 2021 \\
\text { [73] }\end{array}$ \\
\hline Rodent Strain & $\begin{array}{c}\text { M83 A53T Hu aSyn } \\
\text { Mouse }\end{array}$ & $\begin{array}{l}\text { M83 A53T Hu aSyn } \\
\text { Mouse }\end{array}$ & $\begin{array}{l}\text { M83 A53T Hu aSyn } \\
\text { Mouse }\end{array}$ & $\begin{array}{l}\text { GFP-tagged A53T } \\
\text { SNCA KI Mouse }\end{array}$ & $\begin{array}{c}\text { M83 A53T Hu aSyn } \\
\text { Mouse }\end{array}$ & $\begin{array}{c}\text { M83 A53T Hu aSyn } \\
\text { Mouse }\end{array}$ \\
\hline Injectate & Human aSyn & Mouse aSyn & Mouse aSyn & Mouse aSyn & Mouse aSyn & Mouse aSyn \\
\hline Total Dose & $\begin{array}{c}10 \mathrm{ug} \\
\text { (Bilateral) }\end{array}$ & $\begin{array}{l}20 \mathrm{ug} \\
\text { (Bilateral) }\end{array}$ & $\begin{array}{c}10 \mathrm{ug} \\
\text { (Bilateral) }\end{array}$ & $\begin{array}{c}10 \mathrm{ug} \\
\text { (Unilateral) }\end{array}$ & $\begin{array}{c}10 \mathrm{ug} \\
\text { (Bilateral) }\end{array}$ & $\begin{array}{c}20 \mathrm{ug} \\
\text { (Bilateral) }\end{array}$ \\
\hline DPI & $126-160$ & 134 & $30,60,90$ & 120,240 & $30,90,117$ & 45 \\
\hline \begin{tabular}{|l} 
Peripheral/Spinal \\
Cord pS129 aSyn
\end{tabular} & $126+(S C)$ & 134 (SC) & $60+(\mathrm{SC})$ & N/A & N/A & 45 (DRG, SC) \\
\hline CNS pS129 aSyn & $\begin{array}{c}126+\text { (Brainstem, Midbrain, } \\
\text { Forebrain) }\end{array}$ & $\begin{array}{c}134 \text { (Brainstem, Midbrain, } \\
\text { Forebrain) }\end{array}$ & $\begin{array}{c}60+\text { (Brainstem, Midbrain) } \\
90 \text { (Forebrain) }\end{array}$ & $\begin{array}{c}120 \text { (Brainstem) } \\
240 \text { (Brainstem, Midbrain, } \\
\text { Forebrain) }\end{array}$ & N/A & 45 (Brainstem, Forebrain) \\
\hline Cell Loss & N/A & N/A & $60+$ (Motor Neurons) & N/A & N/A & N/A \\
\hline $\begin{array}{l}\text { Behavioral } \\
\text { Deficits }\end{array}$ & Paralysis - $126+$ & Motor - 134 & N/A & N/A & Paralysis - 117 & $\begin{array}{c}\text {-Motor - } 45 \\
\text {-Sensory - } 45\end{array}$ \\
\hline Other & Death - 160 & N/A & $\begin{array}{c}\text {-Ubiquitin and p62 } \\
\text { Inclusions - } 60+ \\
\text {-Astrogliosis - } 90 \\
\text {-Microglial Activation - } 90\end{array}$ & N/A & $\begin{array}{c}\text {-Brain Microstructure } \\
\text { Changes - } 30+ \\
\text {-Reduced Sensory } \\
\text { Activation - } 90 \\
\text {-Reduced Spontaneous } \\
\text { Brain Activity - } 30 \\
\end{array}$ & $\begin{array}{l}\text { Astrogliosis (SC, } \\
\text { Brainstem, Forebrain) - } 45\end{array}$ \\
\hline Note & $\begin{array}{c}\text { Also looked at mutant aSyn } \\
\text { PFFs }\end{array}$ & & Tested lower doses as well & & dMRI and fMRI performed & \\
\hline
\end{tabular}

aSyn, alpha-synuclein; PFFs, preformed fibrils; Hu, human; KI, knockin; SC, spinal cord; DRG, dorsal root ganglia; CNS, central nervous system; dMRI, diffusion magnetic resonance imaging; fMRI, functional magnetic resonance imaging.

Table 9

Injection of aSyn PFFs into the wildtype or transgenic rodent peritoneum, vein, nerve, or eye

\begin{tabular}{|c|c|c|c|c|c|c|c|c|}
\hline \multirow{3}{*}{\begin{tabular}{|c|} 
Paper \\
Rodent Strain
\end{tabular}} & \multirow{2}{*}{\multicolumn{2}{|c|}{$\begin{array}{c}\text { Intraperitoneal } \\
\text { Ayers } 2017 \\
{[70]}\end{array}$}} & \multicolumn{2}{|c|}{ Intravenous } & \multirow{2}{*}{\multicolumn{3}{|c|}{$\begin{array}{c}\text { Nerve } \\
\text { Ayers } 2018 \\
\text { [75] }\end{array}$}} & \multirow{3}{*}{$\begin{array}{c}\text { Intravitreal } \\
\begin{array}{c}\text { Veys 2020 } \\
{[76]}\end{array} \\
\text { C57BI/6 Mouse }\end{array}$} \\
\hline & & & \multirow{2}{*}{$\begin{array}{c}\text { Ayers } 2017 \\
\text { [70] }\end{array}$} & \multirow{2}{*}{$\begin{array}{c}\begin{array}{c}\text { Kuan } 2021 \\
{[74]}\end{array} \\
\begin{array}{c}\text { Sprague Dawley } \\
\text { Rats }\end{array} \\
\end{array}$} & & & & \\
\hline & $\begin{array}{c}\text { M83 A53T Hu aSyn } \\
\text { Mouse }\end{array}$ & $\begin{array}{l}\text { M20 WT Hu aSyn } \\
\text { Mouse }\end{array}$ & & & C57BI/6 Mouse & $\begin{array}{c}\text { M83 A53T Hu aSyn } \\
\text { Mouse }\end{array}$ & $\begin{array}{c}\text { M20 WT Hu aSyn } \\
\text { Mouse }\end{array}$ & \\
\hline Injectate & Mouse aSyn & Mouse aSyn & Mouse aSyn & Human aSyn & Mouse aSyn & Mouse aSyn & Mouse aSyn & Human aSyn \\
\hline Total Dose & 50 ug & 50 ug & 20 ug & 100 ug & 4 ug & 4 ug & 4 ug & 5 ug \\
\hline DPI & 180 & 350 & 120 & 180 & 360 & $30,60,120$ & $60,120,240$ & 90,150 \\
\hline $\begin{array}{l}\text { Peripheral/ } \\
\text { Spinal Cord } \\
\text { pS129 aSyn }\end{array}$ & $180(\mathrm{SC})$ & 350 (SC) & $120(\mathrm{SC})$ & Absent & Absent & $30+(D R G, S C)$ & $\begin{array}{c}60+(\text { SC) } \\
120 \text { (DRG) }\end{array}$ & Absent \\
\hline $\begin{array}{l}\text { CNS pS129 } \\
\text { aSyn }\end{array}$ & $\begin{array}{c}180 \text { (Brainstem, } \\
\text { Midbrain, Forebrain) }\end{array}$ & 350 (Brainstem) & $\begin{array}{c}120 \text { (Brainstem, } \\
\text { Midbrain, Forebrain) }\end{array}$ & Absent & Absent & $\begin{array}{l}60+(\text { Brainstem) } \\
120 \text { (Forebrain) }\end{array}$ & $\begin{array}{c}120+(\text { Brainstem) } \\
\text { Absent (Forebrain) }\end{array}$ & Absent \\
\hline Cell Loss & N/A & N/A & N/A & Absent & N/A & N/A & N/A & $\mathrm{N} / \mathrm{A}$ \\
\hline $\begin{array}{l}\text { Behavioral } \\
\text { Deficits }\end{array}$ & $\begin{array}{l}\text { Motor - } 180 \text { (only in } \\
\text { half of the cohort) }\end{array}$ & Motor - Absent & Motor - Absent & $\begin{array}{c}\text {-Motor - Absent } \\
\text {-GI - Absent } \\
\text {-Olfactory - Absent }\end{array}$ & Absent & Paralysis - 120 & Paralysis - 240 & N/A \\
\hline Other & N/A & $\mathrm{N} / \mathrm{A}$ & N/A & N/A & N/A & $\begin{array}{l}\text { Astrogliosis and } \\
\text { Microgliosis - 60+ } \\
\text { (SC) }\end{array}$ & $\begin{array}{l}\text { Astrogliosis and } \\
\text { Microgliosis - 120+ } \\
\text { (SC) }\end{array}$ & N/A \\
\hline Note & & & & & & & & \\
\hline
\end{tabular}

aSyn, alpha-synuclein; PFFs, preformed fibrils; Hu, human; SC, spinal cord; DRG, dorsal root ganglia; CNS, central nervous system; GI, gastrointestinal.

when analyzing the phenotypes listed in Tables 1-9 when comparing studies of similar designs with regard to injection site, unilateral vs bilateral injection, wildtype vs transgenic rodent, etc.

An example of this can be found in motor deficits observed following intrastriatal injection. Despite using the same dose of aSyn PFFs, some report motor deficits following unilateral intrastriatal injection of mouse aSyn PFFs as early as 90 DPI [28, $30,31]$ while others do not observe motor impairments until 180 DPI $[19,27]$ (Table 1). Others still do not observe motor impairments even at 180 days 

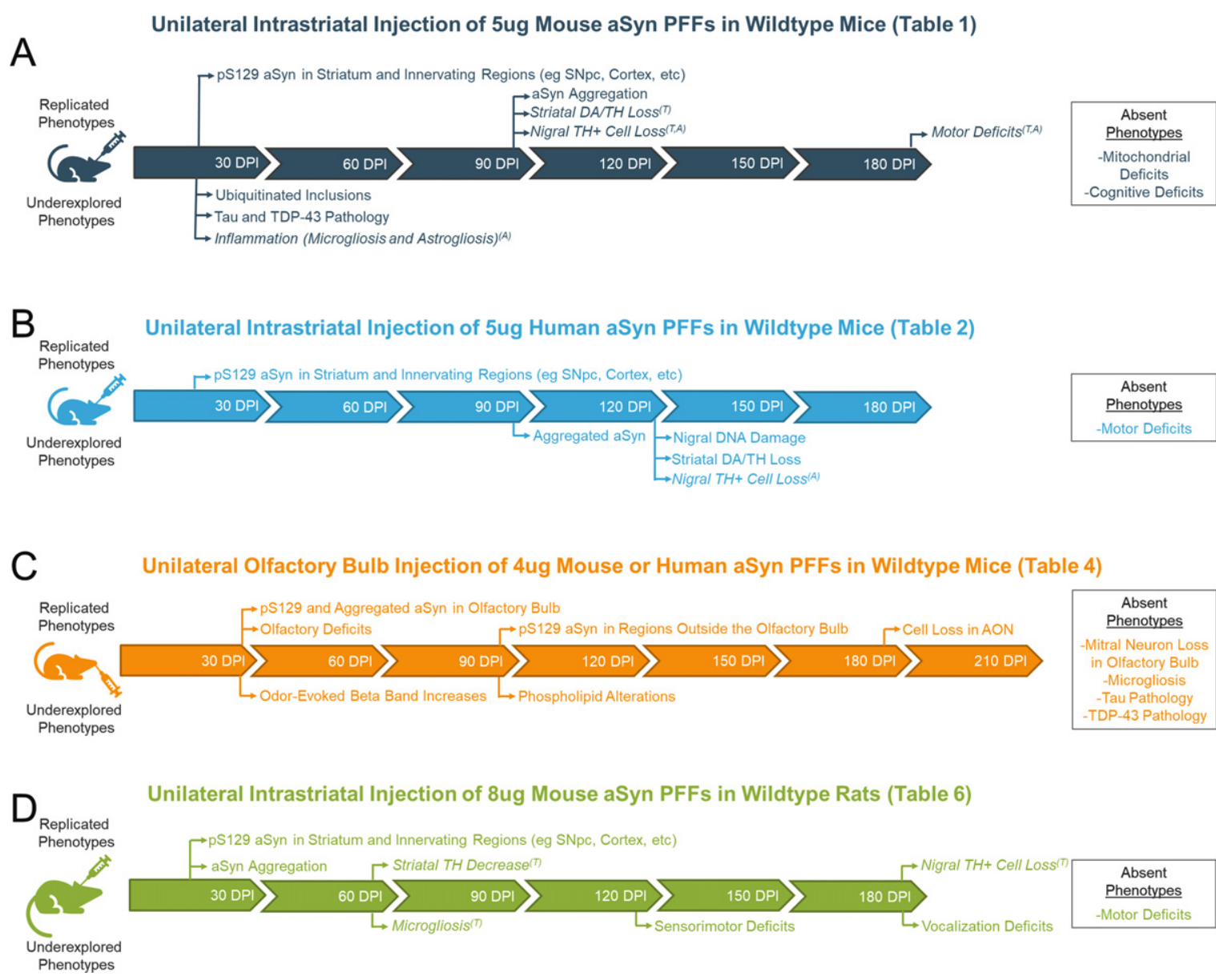

Fig. 1. Visual representation of the various phenotypes reported in common iterations of the alpha-synuclein preformed fibril (aSyn PFF) model. Replicated phenotypes (reported in $>1$ study) and underexplored phenotypes (observed in only 1 study) are mapped across the timeline of the model. Phenotypes that were investigated but found to be absent are also included in an inset to the right of the table. Italicized phenotypes are those that vary across studies by either their presence/absence (denoted by superscript A) or timing of appearance (denoted with superscript T). For all italicized phenotypes, the most common time at which the phenotype is observed is reported.

following bilateral injection [35]. Some of these differences may be attributed to the behavioral assays employed. For instance, Henderson et al. (2019) used two behavioral tests in the same cohort—grip strength and rotarod-and demonstrated differences in grip strength upon aSyn PFF treatment but no effect of aSyn PFF treatment on rotarod performance [30]. These differences in detecting an effect of aSyn PFF treatment on motor function or non-motor function could be due to the physiology probed within these assays, the sensitivity of the tests, or confounds that may impact the readouts [77].

Another phenotype that greatly varies between studies is pS129 aSyn pathology in the brain following injection of aSyn PFFs to the gut (Table 7). Roughly half of the studies observe pS129 aSyn pathology spread to the midbrain/forebrain $[62,64$,
$65,68]$ whereas the other half observe pathology in the periphery/brainstem that never progresses to the midbrain/forebrain [61, 63, 64, 66, 68]. As mentioned in a recent review by Bindas et al. (2021), the reason for this is unclear but could relate to gastrointestinal conditions, amount of pathology generated, site of pathology, and type of pathology induced by the aSyn PFFs [78].

When attempting to understand the variability within the aSyn PFF model, it is important to understand the various factors that can influence the pathogenicity of the aSyn PFFs. Some factors may be obvious and easily accounted for, such as dose or days post-injection. Other factors are not so clear. The source and method of preparing the aSyn PFFs can greatly influence their pathogenicity. Multiple studies have noted that endotoxin may impact the aSyn 
PFF protein $[8,14,78]$. Endotoxin should not only be accounted for due to its ability to generate an immune response that is independent of the aSyn [8, 79], but also for its ability to alter the structure and pathogenicity of the aSyn fibrils themselves [14]. The buffers, temperature, and sonication protocol used to generate aSyn PFFs from monomeric starting material can also lead to variations in the structure of the PFF aggregates that dramatically affect pathogenicity [12-18]. In addition, downstream steps such as storage (duration and temperature) can impact aSyn PFF performance while injection coordinates can impact the pathology observed in the various structures [8].

Taken together, the aSyn PFF model is a popular model due to its ability to recapitulate the pathological hallmarks of PD through the templating of pathology in the endogenous aSyn protein. The model has been used by many to study PD biology and therapeutic interventions targeting aSyn spread, inflammation, neurodegeneration, etc. Although many groups have adopted the model successfully, it is very important to acknowledge the variation in phenotypes between labs. The tables provided in this paper will hopefully assist groups who wish to learn more about the model and clarify which phenotypes are reproducible between labs to prevent issues in adopting the model for one's studies.

\section{ACKNOWLEDGMENTS}

The author would like to sincerely thank Dr. Shalini Padmanabhan at The Michael J. Fox Foundation for Parkinson's Research for her review and comments on this manuscript. The author also would like to thank the Parkinson's disease community of patients and their loved ones for their unending support of the mission of The Michael J. Fox Foundation.

\section{CONFLICT OF INTEREST}

The author has no conflicts of interest to report.

\section{REFERENCES}

[1] Airavaara M, Parkkinen I, Konovalova J, Albert K, Chmielarz P, Domanskyi A (2020) Back and to the future: From neurotoxin-induced to human Parkinson's disease models. Curr Protoc Neurosci 91, e88.

[2] Jagmag SA, Tripathi N, Shukla SD, Maiti S, Khurana S (2016) Evaluation of models of Parkinson's disease. Front Neurosci 9, 503.

[3] Konnova EA, Swanberg M (2018) Chapter 5: Animal models of Parkinson's disease. Parkinson's disease: Pathogenesis and clinical aspects. Codon Publications.
[4] Braak H, Braak E (2000) Pathoanatomy of Parkinson's disease. J Neurol 247(Suppl 2), II3-10.

[5] Braak H, Del Tredici K, Bratzke H, Hamm-Clement J, Sandmann-Keil D, Rub U (2002) Staging of the intracerebral inclusion body pathology associated with idiopathic Parkinson's disease (preclinical and clinical stages). J Neurol 249(Supp 3), III/1-III/5.

[6] Braak, H, Del Tredici J, Rub U, de Vos RAI, Jansen Steur ENH, Braak E (2003) Staging of brain pathology related to sporadic Parkinson's disease. Neurobiol Aging 24, 197-211.

[7] Braak, H, de Vos RAI, Bohl J, Del Tredici K (2006) Gastric alpha-synuclein immunoreactive inclusions in Meissner's and Auerbach's plexuses in cases staged for Parkinson's disease-related brain pathology. Neurosci Lett 396, 67-72.

[8] Polinski NK, Volpicelli-Daley LA, Sortwell CE, Luk KC, Cremades N, Gottler LM, Froula J, Duffy MF, Lee VMY, Martinez TN, Dave KD (2018) Best practices for generating and using alpha-synuclein preformed fibrils to model Parkinson's disease in rodents. J Parkinsons Dis 8, 303-322.

[9] Patterson, JR, Polinski NK, Duffy MF, Kemp CJ, Luk KC, Volpicelli-Daley LA, Kanaan NM, Sortwell CE (2019) Generation of alpha-synuclein preformed fibrils from monomers. $J$ Vis Exp, doi: 10.3791/59758

[10] Zhang B, Kehm V, Gathagan R, Leight SN, Trojanowski JQ, Lee VMY, Luk KC (2019). Stereotaxic targeting of alphasynuclein pathology in mouse brain using preformed fibrils. Methods Mol Biol 1948, 45-57.

[11] Luk KC, Lee VMY (2014) Modeling Lewy pathology propagation in Parkinson's disease. Parkinsonism RelatDisord 20 (Suppl 1(l 1)), S85-87.

[12] Guo JL, Covell DJ, Daniels JP, Iba M, Stieber A, Zhang B, Riddle DM, Kwong LK, Xu Y, Trojanowski JQ, Lee VMY (2013) Distinct alpha-synuclein strains differentially promote tau inclusions in neurons. Cell 154, 103-117.

[13] Peelaerts W, Bousset L, Van der Perren A, Moskalyuk A, Pulizzi R, Giugliano M, Van den Haute C, Melki R, Baekelandt V (2015) Alpha-synuclein strains cause distinct synucleinopathies after local and systemic administration. Nature 522, 340-344.

[14] Kim C, Lv G, Lee JS, Jung BC, Masuda-Suzukake M, Hong CS, Valera E, Lee HJ, Paik SR, Hasegawa M, Masliah E, Eliezer D, Lee SJ (2016) Exposure to bacterial endotoxin generates a distinct strain of alpha-synuclein fibril. Sci Rep 6, 30891 .

[15] Tarutani A, Suzuki G, Shimozawa A, Nonaka T, Akiyama H, Hisanaga SI, Hasegawa M (2016) The effect of fragmented pathogenic alpha-synuclein seeds on prion-like propagation. J Biol Chem 291, 18675-18688.

[16] Abdelmotilib H, Maltbie T, Delic V, Liu Z, Hu X, Fraser KB, Moehle MS, Stoyka L, Anabtawi N, Krendelchtchikova V, Volpicelli-Daley LA, West A (2018) Alpha-synuclein fibril-induced inclusion spread in rats and mice correlates with dopaminergic neurodegeneration. Neurobiol Dis 105, 84-98.

[17] Froula JM, Castellana-Cruz M, Anabtawi NM, Camino JD, Chen SW, Thrasher DR, Freire J, Yazdi AA, Fleming S, Dobson CM, Kumita JR, Cremades N, Volpicelli-Daley LA (2019) Defining alpha-synuclein species responsible for Parkinson's disease phenotypes in mice. J Biol Chem 294, 10392-10406.

[18] Rey NL, Bousset L, George S, Madaj Z, Meyerdirk L, Schulz E, Steiner JA, Melki R, Brundin P (2019) Alphasynuclein conformational strains spread, seed, and target neuronal cells differentially after injection into the olfactory bulb. Acta NeuropatholCommun 7, 221. 
[19] Luk KC, Covell DJ, Kehm VM, Zhang B, Song IY, Byrne MD, Pitkin RM, Decker SC, Trojanowski JQ, Lee VMY (2016) Molecular and biological compatibility with host alpha-synuclein influences fibril pathogenicity. Cell Rep 16, 3373-3387.

[20] Rutherford NJ, Dhillon JKS, Riffe CJ, Howard JK, Brooks M, Giasson BI (2017) Comparison of the in vivo induction and transmission of alpha-synuclein pathology by mutant alpha-synuclein fibril seeds in transgenic mice. Hum Mol Genet 26, 4906-4915.

[21] Guan Y, Zhao X, Liu F, Yan S, Wang Y, Du C, Cui X, Li R, Zhang CX (2020) Pathogenic mutations differentially regulate cell-to-cell transmission of alpha-synuclein. Front Cell Neurosci 14, 159.

[22] Hayakawa H, Nakatani R, Ikenaka K, Aguirre C, Choong CJ, Tsuda H, Nagano S, Koike M, Ikeuchi T, Hasegawa M, Papa SM, Nagai Y, Mochizuki H, Baba K (2020) Structurally distinct alpha-synuclein fibrils induce robust parkinsonian pathology. Mov Disord 35, 256-267.

[23] Luk KC, Kehm VM, Zhang B, O'Brien P, Trojanowski JQ, Lee VMY (2012) Intracerebral inoculation of pathological alpha-synuclein initiates a rapidly progressive neurodegenerative alpha-synucleinopathy in mice. J Exp Med 209, 975-986

[24] Sacino AN, Brooks M, Thomas MS, McKinney AB, Lee S, Regenhardt RW, McGarvey NH, Ayers JI, Notterpek L, Borchelt DR, Golde TE, Giasson BI (2014) Intramuscular injection of alpha-synuclein induces CNS alpha-synuclein pathology and a rapid-onset motor phenotype in transgenic mice. Proc Natl Acad Sci USA 111, 10732-10737.

[25] Terada M, Suzuki G, Nonaka T, Kametani F, Tamaoka A, Hasegawa M (2018) The effect of truncation on prion-like properties of alpha-synuclein. J Biol Chem 293, 1391013920.

[26] Karampetsou M, Ardah MT, Semitekolou M, Polissidis A, Samiotaki M, Kalomoiri M, Majbour N, Xanthou G, ElAgnaf OMA, Vekrellis K (2017) Phosphorylated exogenous alpha-synuclein fibrils exacerbate pathology and induce neuronal dysfunction in mice. Sci Rep 7, 16533.

[27] Luk KC, Kehm V, Carroll J, Zhang B, O'Brien P, Trojanowski JQ, Lee VMY (2012) Pathological alphasynuclein transmission initiates Parkinson-like neurodegeneration in non-transgenic mice. Science 338, 949-953.

[28] Masuda-Suzukake M, Nonaka T, Hosokawa M, Kubo M, Shimozawa A, Akiyama H, Hasegawa M (2014) Pathological alpha-synuclein propagates through neural networks. Acta NeuropatholCommun 2, 88.

[29] Fares MB, Maco B, Oueslati A, Rockenstein E, Ninkina N, Buchman VL, Masliah E, Lashuel HA (2016) Induction of de novo alpha-synuclein fibrillization in a neuronal model for Parkinson's disease. Proc Natl Acad Sci USA 113, E912921.

[30] Henderson MX, Cornblath EJ, Darwich A, Zhang B, Brown H, Gathagan RJ, Sandler RM, Bassett DS, Trojanowski JQ, Lee VMY (2019) Spread of alpha-synuclein pathology through the brain connectome is modulated by selective vulnerability and predicted by network analysis. Nat Neurosci 22, 1248-1257.

[31] Izco M, Blesa J, Verona G, Cooper JM, Alvarez-Erviti L (2021) Glial activation precedes alpha-synuclein pathology in a mouse model of Parkinson's disease. Neurosci Res 170, 330-340

[32] Burtscher J, Copin JC, Sandi C, Lashuel HA (2020) Pronounced alpha-synuclein pathology in a seeding-based mouse model is not sufficient to induce mitochondrial res- piration deficits in the striatum and amygdala. eNeuro 7, ENEURO.0110-20.2020.

[33] Kim YE, Lai TT, Kim YJ, Jeon B (2020) Preferential microglial activation associated with pathological alpha synuclein transmission. J Clin Neurosci 81, 469-476.

[34] Sorrentino ZA, Brooks MMT, Hudson 3rd V, Rutherford NJ, Golde TE, Giasson BI, Chakrabarty P (2017) Intrastriatal injection of alpha-synuclein can lead to widespread synucleinopathy independent of neuroanatomic connectivity. Mol Neurodegener 12, 40.

[35] Stoyka LE, Arrant AE, Thrasher DR, Russell DL, Freire J, Mahoney CL, Narayanan A, Dib AG, Standaert DG, Volpicelli-Daley LA (2020) Behavioral defects associated with amygdala and cortical dysfunction in mice with seeded alpha-synuclein inclusions. Neurobiol Dis 134, 104708.

[36] Ding, XB, Wang XX, Xia DH, Liu H, Tian HY, Fu Y, Chen YK, Qin C, Wang JQ, Xiang Z, Zhang ZX, Cao QC, Wang W, Li JY, Wu E, Tang BS, Ma MM, Teng JF, Wang XJ (2021) Impaired meningeal lymphatic drainage in patients with idiopathic Parkinson's disease. Nat Med 27, 411-418.

[37] Milanese C, Cerri S, Ulusoy A, Gornati SV, Plat A, Gabriels S, Blandini F, Di Monte DA, Joeijmakers JH, Mastroberardino PG (2018) Activation of the DNA damage response in vivo in synucleinopathy models of Parkinson's disease. Cell Death Dis 9, 818.

[38] Earls RH, Menees KB, Chung J, Gutekunst CA, Lee HJ, Hazim MG, Rada B, Wood LB, Lee JK (2020) NK cells clear alpha-synuclein and the depletion of NK cells exacerbates synuclein pathology in a mouse model of alphasynucleinopathy. Proc Natl Acad Sci USA 117, 1762-1771.

[39] Blumenstock S, Rodrigues EF, Peters F, Blazquez-Llorca L, Schmidt F, Giese A, Herms J (2017) Seeding and transgenic overexpression of alpha-synuclein triggers dendritic spine pathology in the neocortex. EMBO Mol Med 9, 716-731.

[40] Bieri G, Brahic M, Bousset L, Couthouls J, Kramer NJ, Ma R, Nakayama L, Monbureau M, Defensor E, Schule B, Shamloo M, Melki R, Gitler AD (2019) LRRK2 modifies alpha-synuclein and spread in mouse models and human neurons. Acta Neuropathol 137, 961-980.

[41] Migdalska-Richards A, Wegrzynowicz M, Harrison IF, Verona G, Bellotti V, Spillantini MG, Schapira AHV (2020) L444P Gba1 mutation increases formation and spread of alpha-synuclein deposits in mice injected with mouse alphasynuclein preformed fibrils. PLoS One 15, e238075.

[42] Rey NL, Steiner JA, Maroof N, Luk KC, Madaj Z, Trojanowski JQ, Lee VMY, Brundin P (2016) Widespread transneuronal propagation of alpha-synucleinopathy triggered in olfactory bulb mimics prodromal Parkinson's disease. J Exp Med 213, 1759-1778.

[43] Rey NL, George S, Steiner JA, Madaj Z, Luk KC, Trojanowski JQ, Lee VMY, Brundin P (2018) Spread of aggregates after olfactory bulb injection of alpha-synuclein fibrils is associated with early neuronal loss and is reduced long term. Acta Neuropathol 135, 65-83.

[44] Graham SF, Rey NL, Ugur Z, Yilmaz A, Sherman E, Maddens M, Bahado-Singh RO, Becker K, Schulz E, Meyerdirk L, Steiner JA, Ma J, Brundin P (2018) Metabolomic profiling of bile acids in an experimental model of prodromal Parkinson's disease. Metabolites 8, 71 .

[45] Kulkarni AS, del Mar Cortijo M, Roberts ER, Suggs TL, Stover HB, Pena-Bravo JI, Steiner JA, Luk KC, Brundin P, Wesson DW(2020) Perturbation of in vivo neural activity following alpha-synuclein seeding in the olfactory bulb. $J$ Parkinsons Dis 10, 1411-1427. 
[46] Uemura N, Ueda J, Yoshihara T, Ikuno M, Uemura MT, Yamakado H, Asano M, Trojanowski JQ, Takahashi R (2021) Alpha-synuclein spread from olfactory bulb causes hyposmia, anxiety, and memory loss in BAC-SNCA mice. Mov Disord, doi: 10.1002/mds.28512

[47] Shen Y, Yu WB, Shen B, Dong H, Zhao J, Tang YL, Fan Y, Yang YF, Sun YM, Luo SS, Chen C, Liu FT, Wu JJ, Xiao BG, Yu H, Koprich JB, Huang ZL, Wang J (2020) Propagated alpha-synucleinopathy recapitulates REM sleep behavior disorder followed by parkinsonian phenotypes in mice. Brain 143, 3374-3392.

[48] Luna E, Decker SC, Riddle DM, Caputo A, Zhang B, Cole T, Caswell C, Xie SX, Lee VMY, Luk KC (2018) Differential alpha-synuclein expression contributes to selective vulnerability of hippocampal neuron subpopulations to fibril-induced toxicity. Acta Neuropathol 135, 855-875.

[49] Nouraei N, Mason DM, Miner KM, Carcella MA, Bhatia TN, Dumm BK, Soni D, Johnson DA, Luk KC, Leak RK (2018) Critical appraisal of pathology transmission in the alpha-synuclein fibril model of Lewy body disorders. Exp Neurol 299(Pt A), 172-196.

[50] Caputo A, Liang Y, Raabe TD, Lo A, Horvath M, Zhang B, Brown HJ, Stieber A, Luk KC (2020) SNCA-GFP knock-in mice reflect patterns of endogenous expression and pathological seeding. eNeuro 7, ENEURO.0007-20.2020.

[51] Osterberg VR, Spinelli KJ, Weston LJ, Luk KC, Woltjer RL, Unni VK (2015) Progressive aggregation of alpha-synuclein and selective degeneration of Lewy inclusion-bearing neurons in a mouse model of parkinsonism. Cell Rep 10, 1252-1260.

[52] Schaser AJ, Stackhouse TL, Weston LJ, Kerstein PC, Osterberg VR, Lopez CS, Dickson DW, Luk KC, Meshul CK, Woltjer RL, Unni VK (2020) Trans-synaptic and retrograde axonal spread of Lewy pathology following preformed fibril injection in an in vivo A53T alpha-synuclein mouse model of synucleinopathy. Acta NeuropatholCommun 8, 150.

[53] Masuda-Suzukake M, Nonaka T, Hosokawa M, Oikawa T, Arai T, Akiyama H, Mann DMA, Hasegawa M(2013) Prionlike spreading of pathological alpha-synuclein in brain. Brain 136, 1128-1138.

[54] Paumier KL, Luk KC, Manfredsson FP, Kanaan NM, Lipton JW, Collier TJ, Steece-Collier K, Kemp CJ, Celano S, Schulz E, Sandoval IM, Fleming S, Dirr E, Polinski NK, Trojanowski JQ, Lee VMY, Sortwell CE (2015) Intrastriatal injection of preformed mouse alpha-synuclein fibrils into rats triggers alpha-synuclein pathology and bilateral nigrostriatal degeneration. NeurobiolDis 82, 185-199.

[55] Duffy MF, Collier TJ, Patterson JR, Kemp CJ, Luk KC, Tansey MG, Paumier KL, Kanaan NM, Fischer DL, Polinski NK, Barth OL, Howe JW, Vaikath NN, Majbour NK, ElAgnaf OMA, Sortwell CE (2018) Lewy body-like alphasynuclein inclusions trigger reactive microgliosis prior to nigral degeneration. J Neuroinflammation 15, 129.

[56] Patterson JR, Duffy MF, Kemp CJ, Howe JW, Collier TJ, Stoll AC, Miller KM, Patel P, Levine N, Moore DJ, Luk KC, Fleming SM, Kanaan NM, Paumier KL, El-Agnaf OMA, Sortwell CE (2019) Time course and magnitude of alpha-synuclein inclusion formation and nigrostriatal degeneration in the rat model of synucleinopathy triggered by intrastriatal alpha-synuclein preformed fibrils. Neurobiol Dis 130, 104525.

[57] Thomsen MB, Ferreira SA, Schacht AC, Jacobsen J, Simonsen M, Betzer C, Jensen PH, Brooks DJ, Landau AM, Romero-Ramos M (2021) PET imaging reveals early and progressive dopaminergic deficits after intra-striatal injec- tion of preformed alpha-synuclein fibrils in rats. Neurobiol Dis 149, 105229.

[58] Creed RB, Goldberg MS (2020) Enhanced susceptibility of PINK1 knockout rats to alpha-synuclein fibrils. Neuroscience $\mathbf{4 3 7}, 64-75$.

[59] Thakur P, Breger LS, Lundblad M, Wan OW, Mattsson B, Luk KC, Lee VMY, Trojanowski JQ, Bjorklund A (2017) Modeling Parkinson's disease pathology by combination of fibril seeds and alpha-synuclein overexpression in the rat brain. Proc Natl Acad Sci USA 114, E8284-E8293.

[60] Harms AS, Delic V, Thome AD, Bryant N, Liu Z, Chandra S, Jurkuvenaite A, West AB (2017) Alpha-synuclein fibrils recruit peripheral immune cells in the rat brain prior to neurodegeneration. Acta NeuropatholCommun 5, 85.

[61] Uemura N, Yagi H, Uemura MT, Hatanaka Y, Yamakado $\mathrm{H}$, Takahashi R (2018) Inoculation of alpha-synuclein preformed fibrils into the mouse gastrointestinal tract induces Lewy body-like aggregates in the brainstem via the vagus nerve. Mol Neurodegener 13, 21.

[62] Kim S, Kwon SH, Kam TI, Panicker N, Karuppagounder SS, Lee S, Lee JH, Kim WR, Kook M, Foss CA, Shen C, Lee H, Kulkarni S, Pasricha PJ, Lee G, Pomper MG, Dawson VL, Dawsom TM, Ko HS (2019) Transneuronal propagation of pathologic alpha-synuclein from the gut to the brain models Parkinson's disease. Neuron 103, 627-641.

[63] Uemura N, Yagi H, Uemura MT, Yamakado H, Takahashi R (2020) Limited spread of pathology within the brainstem of alpha-synuclein BAC transgenic mice inoculated with preformed fibrils into the gastrointestinal tract. Neurosci Lett 716, 134651.

[64] Challis C, Hori A, Sampson TR, Yoo BB, Challis RC, Hamilton AM, Mazmanian SK, Volpicelli-Daley LA, Vradinaru V (2020) Gut-seeded alpha-synuclein fibrils promote gut dysfunction and brain pathology specifically in aged mice. Nat Neurosci 23, 327-336.

[65] Wang XJ, Ma MM, Zhou LB, Jiang XY, Hao MM, Teng RKF, Wu E, Tang BS, Li JY, Teng JF, Ding XB (2020) Autonomic ganglionic injection of alpha-synuclein fibrils as a model of pure autonomic failure alpha-synucleinopathy. Nat Commun 11, 934.

[66] Holmqvist S, Chutna O, Bousset L, Aldrin-Kirk P, Li W, Bjorklund T, Wang ZY, Roybon L, Melki R, Li JY (2014) Direct evidence of Parkinson pathology spread from the gastrointestinal tract to the brain in rats. Acta Neuropathol 128, 805-820.

[67] Manfredsson FP, Luk KC, Benskey MJ, Gezer A, Garcia J, Kuhn NC, Sandoval IM, Patterson JR, O'Mara A, Yonkers R, Kordower JH (2018) Induction of alpha-synuclein pathology in the enteric nervous system of the rat and non-human primate results in gastrointestinal dysmotility and transient CNS pathology. Neurobiol Dis 112, 106-118.

[68] Van Den Berge N, Ferreira N, Gram J, Mikkelsen TW, Alstrup AKO, Casadei N, Tsung-Pin P, Riess O, Nyengaard JR, Tamguney G, Jensen PH, Borghammer P (2019) Evidence for bidirectional and trans-synaptic parasympathetic and sympathetic propagation of alpha-synuclein in rats. Acta Neuropathol 138, 535-550.

[69] Van Den Berge N, Ferreira N, Mikkelsen TW, Alstrup AKO, Tamguney G, Karlsson P, Terkelsen AJ, Nyengaard JR, Jensen PH, Borghammer P (2021) Ageing promotes pathological alpha-synuclein propagation and autonomic dysfunction in wild-type rats. Brain 144, 1853-1868.

[70] Ayers JI, Brooks MM, Rutherford NJ, Howard JK, Sorrentino ZA, Riffe CJ, Giasson BI (2017) Robust central nervous system pathology in transgenic mice following 
peripheral injection of alpha-synuclein fibrils. J Virol 91, e02095-16.

[71] Sorrentino ZA., Xia Y, Funk C, Riffe CJ, Rutherford NJ, Diaz CC, Sacino AN, Price ND, Golde TE, Giasson BI, Chakrabarty P (2018) Motor neuron loss and neuroinflammation in a model of alpha-synuclein-induced neurodegeneration. Neurobiol Dis 120, 98-106.

[72] Chu WT, DeSimone JC, Riffe CJ, Liu H, Chakrabarty P, Giasson BI, Vedam-Mai V, Vaillancourt DE (2020) Alpha-synuclein induces progressive changes in brain microstructure and sensory-evoked brain function that precedes locomotor decline. J Neurosci 40, 6649-6659.

[73] Ferreira N, Goncalves NP, Jan A, Moller Jensen N, van der Laan A, Mohseni S, Vaegter CB, Jensen PH(2021) Transsynaptic spreading of alpha-synuclein pathology through sensory afferents leads to sensory nerve degeneration and neuropathic pain. Acta NeuropatholCommun 9, 31.

[74] Kuan WL, Stott K, He X, Wood TC, Yang S, Kwok JCF, Hall K, Zhao Y, Tietz O, Aigbirhio FI, Vernon AC, Barker RA (2021) Systemic alpha-synuclein injection triggers selective neuronal pathology as seen in patients with Parkinson's disease. Mol Physiol 26, 556-567.
[75] Ayers JI, Riffe CJ, Sorrentino ZA, Diamond J, Fagerli E, Brooks M, Galaleldeen A, Hart PJ, Giasson BI (2018) Localized induction of wild-type and mutant alpha-synuclein aggregation reveals propagation along neuroanatomical tracts. J Virol 92, e00586-18.

[76] Veys L, Van Houcke J, Aerts J, Van Pottelberge S, Mahieu M, Coens A, Melki R, Moechars D, De Muynck L, De Groef L (2021) Absence of uptake and prion-like spreading of alpha-synuclein and tau after intravitreal injection of preformed fibrils. Front Aging Neurosci 12, 614587.

[77] Brooks SP, Dunnett SB (2009) Tests to assess motor phenotype in mice: a user's guide. Nat Rev Neurosci 10, 519-529.

[78] Bindas AJ, Kulkarni S, Koppes RA, Koppes AN (2021) Parkinson's disease and the gut: models of an emerging relationship. Acta Biomater, doi: 10.1016/j.actbio.2021.03.071

[79] Urbaschek B, Urbaschek R (1979) The inflammatory response to endotoxin. BiblAnat 17, 74-104. 